\title{
New Tools for Visualization and Quantification in Dynamic Processes: Application to the Nuclear Envelope Dynamics During Mitosis
}

\author{
J. Mattes ${ }^{1}$, J. Fieres ${ }^{1}$, J. Beaudouin ${ }^{2}$, D. Gerlich ${ }^{1}$, J. Ellenberg ${ }^{2}$, and R. Eils ${ }^{1}$ \\ 1 iBioS, DKFZ Heidelberg, 69120 Heidelberg, Germany \\ J.Mattes@dkfz.de \\ 2 EMBL, Heidelberg, Germany
}

\begin{abstract}
The aim of this paper is to present new tools for visualization and quantification in dynamic processes. We concentrate on the concise description of local quantitative values and on a easy-to-apprehend way to visualize them. Our approach is based on registration in order to obtain a transformation for the whole image space. This transformation permits us to obtain local values such as bending or velocity in each pixel. We define a density function using this values and apply confinement tree analysis on them. We present an application in cell biology where we can obtain new insight into the elasticity properties of the nuclear lamina.
\end{abstract}

\section{Introduction}

Modern imaging devices make it possible to study in vivo the mechanism of dynamics in living systems. Intra-operative MRI devices producing several images per second, offer nowadays the possibility to follow the heart-cycle in realtime. Tumor growth can be supervised by imaging systems guiding the focus of a radiation source. At a lower scale, fluorescent confocal microscopy permits to observe marked cellular structures such as chromatin or the nuclear envelope over time.

In order to quantitatively analyze and to visualize the movements of objects in the image time series in an easy-to-apprehend way sophisticated computer algorithms are required [1]. A difficulty is a concise quantitative description permitting the comparision of observed motion in different images going beyond the estimation of the velocity vector field. In this paper, we focus on the description and quantification of local deformation and we let aside considerations about the fit of the observed movement with global reference dynamics [3].

\section{Methods}

Our approach to analyze deformation in the local spatial domain is based on registration in the image time series and on a statistical analysis, namely the confinement tree analysis (CTA) [4, of the local parameters obtained as a result of the registration step. In a first preprocessing step (i), we extract points describing representative structures of the image, eventually after the application 
of a filtering operator. In the second step (ii), we register consecutive images based on the extracted point sets. The registration algorithm comprises an error functional, an optimization strategy and a motion model and is detailed in [2]. The error functional is defined using the extracted image structures. The motion model is necessary as the optimization problem is ill posed in order to restrict the solution space and to model the allowed volume transformations. It is determined by the initial position of several freely adjustable control points, by the use of thin plate splines to interpolate between them, and by an additional regularization term in the error functional. The obtained transformation permits us, in the third step (iii), to calculate local quantitative values for each pixel such as velocity, strain, or bending. We represent the absolute values obtained for each pixel as an intensity value and produce a new image. Finally, in the fourth step (iv), we apply CTA on the new image as a statistical method to describe the deformation concisely. Given an intensity function $f: \mathbb{R}^{d} \rightarrow \mathbb{R}_{\geq 0}, d=2,3$, the confiners are defined as the maximal connected subsets (i.e., components) $\mathcal{C}_{l}$ of the level sets $\mathcal{L}_{l}=\left\{x \in \mathbb{R}^{d} \mid f(x) \geq l\right\}, l \in \mathbb{R}_{\geq 0}$. Considering them taken on several levels $l_{k}, k=1, \ldots, r$ including the 0 level, they define obviously a tree (by "set inclusion") which we will call confinement tree 4]. Movements in different images can be compared by comparing corresponding confiners. The area of the confiners indicates to what degree the movement is (homogeneously) directed.

\section{Application, Conclusions, and Further Work}

We present an application in cell biology where the dynamics of the nuclear envelope during mitosis is investigated. A $4 \mathrm{D}$ sequence of fluorescently labeled (by GFP fusion proteins) lamina is acquired with a confocal laser scanning microscop. We obtain one 3D image every 10-30 seconds. We will focus on the formation of holes in the nuclear envelope during its breakdown taking place in prometaphase. To determine deformation in the upper surface of the lamina, artificial landmarks were introduced by photobleaching of a grid.

The upper row in Figure 1 shows the slice representing at best the upper side of the nuclear envelope and the evolution of the bleached grid during time. The confiner contours of the corresponding velocity intensity images shown in the lower row are superimposed. Interestingly, the black area in the middle of image $f$ coincides perfectly with the hole emerging between images b and c. Thus, there is no movement at the emerging hole itself but a strong and homogeneous movement adjacent to it and directed away from it. If we imagine the lamina in this situation as an elastic polymer under tension it appears normal that an emerging hole causes this kind of dynamics. The precise coincidence illustrates also how reliable our algorithm estimates the underlying motion. We observe a movement towards the hole of the upper confiner in image $g$ and for the confiner in the lower right corner. It seems to represent a back coupling of the movements before and to illustrate vaccilation properties of the lamina. The average area of the selected confiners assesses the movement in image h as highly undirected. This could correspond to the end of a vaccilation-relaxation process. 


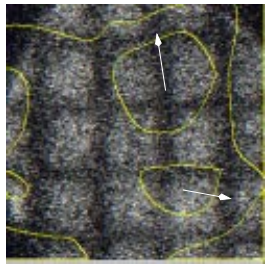

a

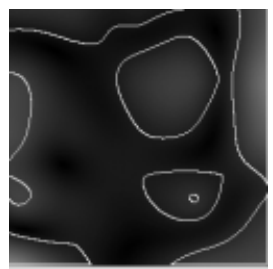

$\mathrm{e}$

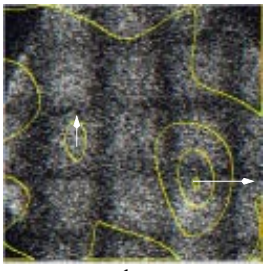

b

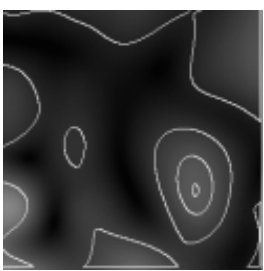

$\mathrm{f}$

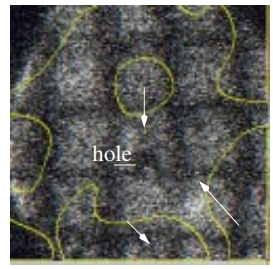

$\mathrm{c}$

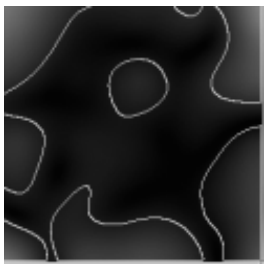

g

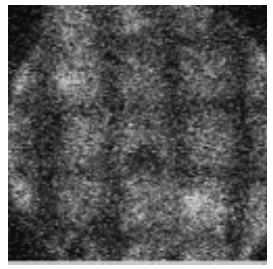

d

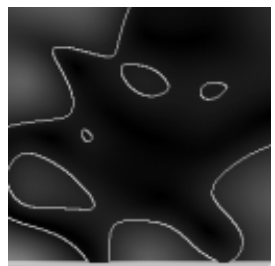

$\mathrm{h}$

Fig. 1. Formation of a hole (images $c$ and $d$, indicated in $c$ ) in the lamina and velocity analysis: images a-d show the evolution of the photobleached grid during time with the superimposed confiner contours of the corresponding velocity intensity image depicted in e-h (image e corresponds to images a and $b$, image $f$ to the images $b$ and $c$, etc.; image $h$ corresponds to $d$ and the following image in the time sequence which is not shown). The arrows show the average direction of the movement inside a confiner.

We described, characterized and visualized complex local dynamics by a few image clusters of homogeneous motion connected by a tree structure allowing new ways to analyze them quantitatively. In the presented cell biological application we thus obtained new insight into the physical properties of the lamina and could establish a correlation between surface movement and the formation of a hole in it. In further work we will investigate the possibility to compare local dynamics with reference dynamics, for instance with membrane vaccilations. In long term, we will design a model reproducing the movements of a polymer during the formation of a hole consistently with the imaged data.

\section{References}

1. Ferrant, M., Nabavi, A., Kikinis, R., Warfield, S.K.: Real-time simulation and visualization of volumetric brain deformation for image-guided neurosurgery. SPIE Medical Imaging 2001 (in press)

2. Fieres, J., Mattes, J., Eils, R.: A point set registration algorithm using a motion model based on thin-plate splines and point clustering. DAGM 2001, LNCS, Springer Verlag (accepted)

3. Germain, F., Doisy, A., Ronot, X., Tracqui, P.: Characterization of cell deformation and migration using a parametric estimation of image motion IEEE Trans. Biomed. Engineering 46 (1999) 584-600

4. Mattes, J., Demongeot, J.: Tree representation and implicit tree matching for a coarse to fine image matching algorithm. In: MICCAI'99, C. Taylor, A. Clochester (Eds.). LNCS. Springer-Verlag (1999) 646-655 